\title{
Critical packing in granular shear bands
}

\author{
S. Fazekas ${ }^{1,2}$, J. Török ${ }^{1}$ and J. Kertész ${ }^{1}$ \\ ${ }^{1}$ Department of Theoretical Physics, \\ ${ }^{2}$ Theoretical Solid State Research Group of the Hungarian Academy of Sciences, \\ Budapest University of Technology and Economics (BME), \\ H-1111 Budapest, Hungary
}

(Dated: October 13, 2006)

\begin{abstract}
In a realistic three-dimensional setup, we simulate the slow deformation of idealized granular media composed of spheres undergoing an axisymmetric triaxial shear test. We follow the selforganization of the spontaneous strain localization process leading to a shear band and demonstrate the existence of a critical packing density inside this failure zone. The asymptotic criticality arising from the dynamic equilibrium of dilation and compaction is found to be restricted to the shear band, while the density outside of it keeps the memory of the initial packing. The critical density of the shear band depends on friction (and grain geometry) and in the limit of infinite friction it defines a specific packing state, namely the dynamic random loose packing.
\end{abstract}

PACS numbers: 45.70.Cc, 81.40.Jj

Keywords: granular compaction, stress-strain relation

\section{INTRODUCTION}

Packing density of different particulate systems is of main interest for scientific fields including, but not limited to, suspensions, metallic glasses, molecular systems, and granular materials. In three dimensions, for identical spheres, the face-centered cubic (FCC) packing is the maximum possible [1]. This fills the space with a volume fraction of $\pi /(3 \sqrt{2}) \approx 0.74$. Random arrangements have much lower densities 2]. Different experiments and computer simulations revealed that the largest obtainable volume fraction of a random packing of identical spheres is around 0.64. This is known as the random close packing (RCP) limit. A mathematical definition of this limit can be given through the concept of maximally random jammed state [3, 4].

Reaching the RCP limit needs careful preparation (e.g. tapping and compression). If glass or marble beads are simply poured into a container the volume fraction is usually only around 0.6. A random loose packing (RLP) at its limit of mechanical stability obtained by immersing spheres in a fluid and letting them settle has a volume fraction of 0.555 [5]. The volume fraction of RLPs obtained with different methods (both experimental and numerical) show that this packing state is less well defined than the RCP limit. Attempts made in order to relate RLP to rigidity percolation [5] and to critical density at jamming of an assembly of (infinitely) rough spheres [6], are to be mentioned.

Already in 1885, Reynolds noted that dense granular samples dilate during slow deformation [7]. On the other hand, it is well known that loose granular materials densify in such a process [8, 9]. Under slow shear the strain is usually localized to narrow domains called shear bands. As it was first suggested by Casagrande [10], it is tempting to assume that in these failure zones the system selforganizes its packing density to a critical value independent of the initial packing state of the material.
While this hypothesis forms the basis of many continuum constitutive models of soil mechanics since decades [1], a general micromechanical theory of shear band formation and of the involved criticality is still missing. Progress, needed in order to deepen our understanding of the critical state in shear bands, can be expected from the remarkable development of experimental techniques (including Computer Tomography [12, 13] and measurements in microgravity [14, 15]) and of simulation techniques which become increasingly efficient as computational power grows [16].

Shearing of granular materials has been investigated in many different geometries and specially designed laboratory tests (for recent results see [12, 14]). Such experimental studies revealed complex localization patterns and presented evidence for the existence of a critical particle density inside the shear bands. The importance of computer simulations is enhanced by the fact that they make possible studies which are difficult to control in experiments (e.g. friction dependence) and they facilitate the measurement of hardly accessible quantities (e.g. volume fraction inside the shear bands).

The critical density, in numerical studies, is often studied only in special conditions when shearing extends to the whole volume of the samples. This allowed for discussing the criticality based simply on global behavior (e.g. dilatancy). Without reference to shear bands, many qualitative effects were already pointed out in both mathematical models [17, 18] and simulations [18, 19]. However, such studies neglected the involved localization phenomena inevitable in real situations and disregarded the self-organizing manner in which the packing state of the shear bands is usually formed.

A principal parameter which controls the dynamic equilibrium between dilation and compaction in fully developed shear bands is the friction between the grains. Intuitively, a system of frictionless grains can be sheared at a large packing density (close to the RCP limit) because 


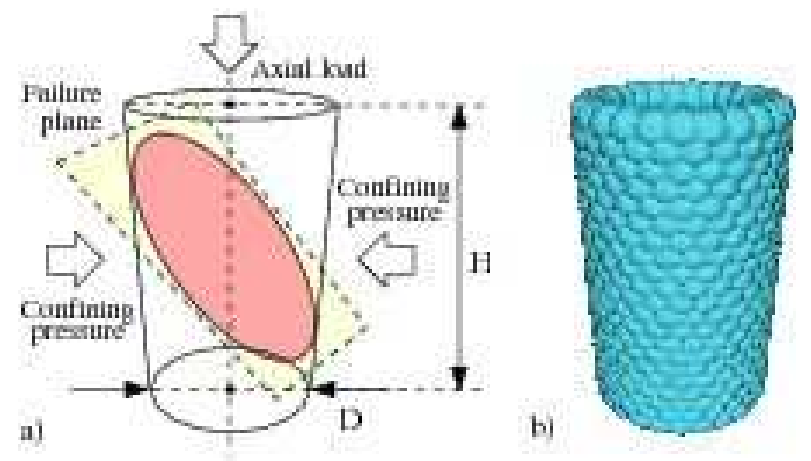

FIG. 1: (Color online) a) Grains placed between two horizontal platens and surrounded by an elastic membrane were subjected to a vertical load and a lateral confining pressure. b) The membrane was modeled with overlapping spheres [20].

the grains (under slow shear) can easily rearrange in compact configurations. At large friction the rearrangement of the grains is hindered by friction, consequently the packing density of the shear bands is expected to define a low density state close to the RLP limit.

The aim of this Paper is to study the emergence of a critical packing state in sheared granular media and to present its relation to shear bands as well as its dependence on friction.

\section{SIMULATIONS}

We investigate numerically an axisymmetric triaxial shear test (see Fig. 1). This consists of the slow compression of a cylindrical sample enclosed between two end platens. The sample is surrounded by an elastic membrane on which an external confining pressure is applied. The end platens are pressed against each other in a strain controlled way. The upper platen is allowed to tilt. In certain conditions a planar shear band is formed [12]. Using different initial packing densities and friction properties of the grains as well as identifying the grains in the failure zones makes it possible to study the critical packing density inside the shear bands.

The simulations, which are going to be presented here, are based on a standard three-dimensional Distinct Element Method (DEM) 21]. We implemented the Hertz contact model 22] with appropriate damping combined with a frictional spring-dashpot model [16]. The normal $F_{n}$ and the tangential $\mathbf{F}_{t}$ forces are calculated as

$$
\begin{aligned}
F_{n} & =\kappa_{n} \delta_{n}^{3 / 2}-\gamma_{n} \delta_{n}^{1 / 2} v_{n} \\
\mathbf{F}_{t} & =\kappa_{t} \boldsymbol{\delta}_{t}-\gamma_{t} \mathbf{v}_{t}
\end{aligned}
$$

where $\kappa_{n}=10^{6} \mathrm{~N} / \mathrm{m}^{3 / 2}, \kappa_{t}=10^{4} \mathrm{~N} / \mathrm{m}, \gamma_{n}=1 \mathrm{~N} \mathrm{~s} / \mathrm{m}^{3 / 2}$, and $\gamma_{t}=1 \mathrm{~N} \mathrm{~s} / \mathrm{m}$ are normal and tangential stiffness and damping coefficients, $\delta_{n}$ and $\boldsymbol{\delta}_{t}$ are normal and tangential displacements, and $v_{n}$ and $\mathbf{v}_{t}$ are the normal and tangential relative velocities.

\begin{tabular}{c|cccccc}
$\mu_{0}$ & 0.8 & 0.5 & 0.3 & 0.2 & 0.1 & 0.0 \\
\hline$\eta_{0}$ & 0.555 & 0.562 & 0.578 & 0.599 & 0.621 & 0.641
\end{tabular}

TABLE I: Volume fraction $\eta_{0}$ of samples prepared with different coefficients of friction $\mu_{0}$. With each $\mu_{0}$ we prepared 2 samples having the same $\eta_{0}$ within $0.2 \%$ relative error.

The numerical values are chosen to realize the hardest material that we could safely simulate with the minimal damping preserving the numerical stability of the calculations. With the above stiffness and damping coefficients, the inverse of the average eigenfrequency of contacts, in both normal and tangential direction, is more than one order of magnitude larger than the used integration time step $\Delta t=10^{-6} \mathrm{~s}$. This assured that the noise level induced by numerical errors is kept low.

With relatively small samples (made up of 27000 particles) but in a realistic geometry we have succeeded to reproduce shear band morphologies [20, 23] known from experiments [12, 14]. In order to study the criticality of these shear bands, we prepared homogeneous initial configurations of different volume fractions using the deposition method described in [20].

We used a particle distribution similar to those encountered in experimental studies of idealized granular materials. Our particles are spherical, they have equal mass density $\left(2.5 \cdot 10^{3} \mathrm{~kg} / \mathrm{m}^{3}\right)$, equal friction coefficient, and their diameters are set according to a narrow Gaussian distribution with mean $d=0.9 \mathrm{~mm}$ and standard deviation of $2.77 \%$. The prepared cylindrical samples, having diameter $D=23.3 d$, consisted of 20000 to 27000 spherical grains as required by a prescribed packing density and the $H \approx 2.2 \mathrm{D}$ geometrical constraint, where $H$ is the height of the samples.

Initially the particles were placed randomly in a tall cylinder (about 3 times taller than $H$ ). They were given small downwards velocities in such a way that they all collided approximately at the same time. The upper platen was pressed on top of the packing to hold it together. This method provides an efficient way to produce a homogeneous random packing. The volume fraction of the prepared samples could be controlled in the full RLP to RCP range (see Tab. I) by varying the coefficient of friction $\mu_{0}$ which was applied during this phase.

After preparation, the friction coefficient of the particles was set to a new value $\mu$ independent of $\mu_{0}$. During the simulations, similarly to [20], we compressed the samples vertically at zero gravity and $0.5 \mathrm{kPa}$ confining pressure. The bottom platen was fixed. The upper platen moved downward with a constant velocity, inducing an axial strain rate of $20 \mathrm{~mm} / \mathrm{s}$. During compression the upper platen could freely tilt along any horizontal axis with rotational inertia $I=10^{-7} \mathrm{~kg} \mathrm{~m}^{2}$.

The lateral membrane surrounding the sample was modeled with approximately 15000 identical, overlapping, non-rotating, frictional spheres connected with elastic springs. The stiffness of the springs was set to 
$\kappa_{s}=0.5 \mathrm{~N} / \mathrm{m}$. This prevented the particles from escaping by passing through the membrane. The membrane particles were initially arranged in a triangular lattice (Fig. 1 (b)). The confining pressure was applied on the triangular facets formed by the neighboring "membrane nodes" as described in [20] (see also [24, 25, 26]).

It is worth mentioning that Cui and O'Sullivan [26] have introduced a technique which speeds up calculations by computing only a section of the cylindrical sample. This allows for larger samples but requires that the symmetry of the system is kept during compression, and thus eliminates the possibility of symmetry breaking strain localization, which arises spontaneously [20] if tilting of the upper platen is not suppressed (see Fig. 1 (a)).

We have executed several simulation runs. The grainplaten and grain-membrane contacts were calculated similarly to grain-grain contacts including the friction properties. The samples of different initial volume fractions (see Tab. 【) were first compressed using the same coefficient of friction $\mu=0.5$. Later, we compressed the densest samples $\left(\eta_{0}=0.641\right)$ with 10 different friction coefficients $\mu \in\{0,0.1, \ldots 0.9\}$. For each set of parameters, two simulation runs were executed using specimens prepared with different random seeds.

During compression, we measured locally the shear intensity $S$ and the volume fraction $\eta$. The regular triangulation of the spherical grains [27, 28] was used to define these quantities. The local volume fraction is given by the ratio of the volumes of a grain and its regular Voronoi cell. The local shear intensity is calculated from the macroscopic strain tensor derived from particle displacements [20, 29]. Using the eigenvalues $\varepsilon_{k}$ of this tensor, we defined the local shear intensity as

$$
S=\max _{k}\left|\varepsilon_{k}-\frac{1}{3} \sum_{l} \varepsilon_{l}\right| .
$$

To overcome fluctuations due to random packing and rearrangements, we calculated spatial averages up to 3rd order neighbors along the regular triangulation.

\section{IDENTIFICATION OF HIGH SHEAR INTENSITY REGIONS}

Strain localization in dense and loose samples shows substantial differences [12]. In dense samples shear bands are usually formed after a short plastic deformation and inside them the local packing density is lower than in the bulk (i.e. the regions outside of the shear bands). Since denser parts are more stable, the position of the shear bands remains unchanged for the whole duration of a shear test. Contrary, in loose samples the shear bands have a slightly higher packing density than the bulk and hence the position of the shear bands is likely to change and to move around the whole sample. This leads to more or less homogeneous samples with local packing densities close to the packing density of the shear bands.
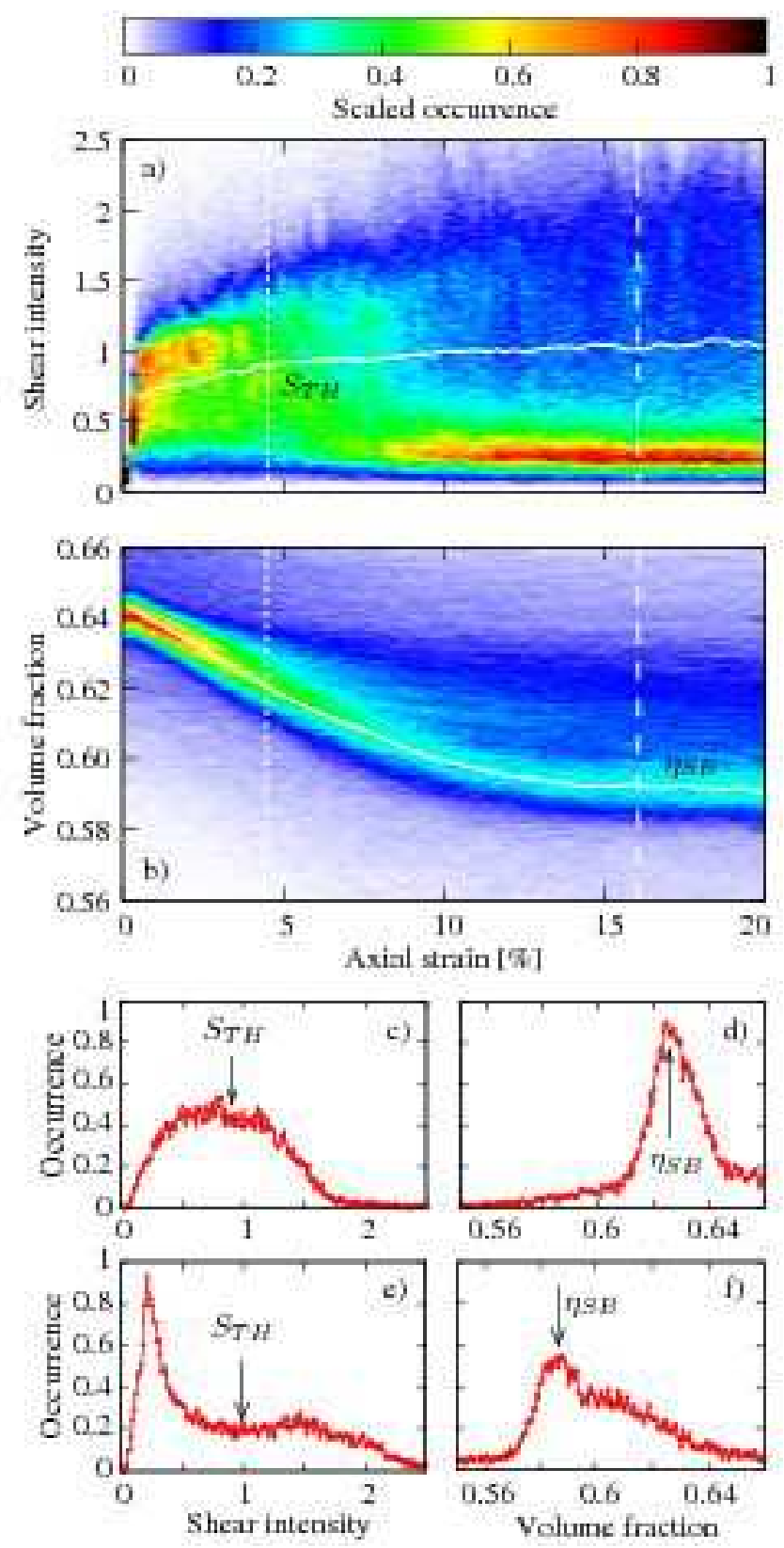

FIG. 2: (Color online) Example of shear intensity and volume fraction histogram maps $\left(\eta_{0}=0.641, \mu=0.5\right)$. The shear intensity is measured in arbitrary units. On $(\mathrm{a}, \mathrm{b})$ the occurrences (scaled to $[0,1])$ are encoded with the color scale shown at the top. On (a) the white curve marks the shear intensity threshold $S_{T H}$. On (b) it marks the shear band volume fraction $\eta_{S B}$. The dotted and dashed vertical lines on $(\mathrm{a}, \mathrm{b})$ at $4.4 \%$ and $16 \%$ axial strain mark the position of the histograms (c, d) and (e, f), respectively.

The general algorithmic identification of failure zones based on geometric methods is difficult, especially, regarding the identification of the non-persistent shear bands of the loose samples. Nevertheless, based on the calculated local shear intensity, individual grains can be categorized to be part of the failure zones or the bulk 
providing that a good enough threshold separating these two classes can be found. A histogram technique seems to be a perfect candidate for this.

Fig. 2 presents histograms of the local shear intensity and local volume fraction. It shows the main aspects of the strain localization process observed in one of our simulations executed with a sample having $\eta_{0}=0.641$ and $\mu=0.5$. At the beginning of the test, up to approximately $6 \%$ axial strain, in shear intensity histograms (see Fig. 2 (a, c)), we find a single peak at medium $S$. This means that almost all particles rearrange simultaneously and consequently the sample experiences a more or less plastic deformation. This is underlined by the fact that the local volume fraction has just one strong peak (Fig. 2 (d)), indicating that the sample is still homogeneous.

At higher $(>6 \%)$ axial strain a shear band is formed. This is localized to a planar failure zone of width of approximately 10 particle diameters and is characterized by much higher $S$ than the bulk. In the bulk the shear intensity fluctuations are small, while these fluctuations are large in the shear band. Consequently, in shear intensity histograms (Fig. 2 (e)), we find a narrow peak at low $S$, corresponding to the bulk, and a wide peak at high $S$, corresponding to the shear band. The volume fraction histogram does also become more structured showing evidence of a non-homogeneous material. The narrow peak at low volume fraction corresponds to the shear band while the bulk produces a much wider distribution at a higher volume fraction (Fig. 2 (f)).

Motivated by this separation, we computed a shear intensity threshold $S_{T H}$, which could be used to define two classes of shear intensity values (low and high) and to classify the grains accordingly into shear band and bulk. For this we used Otsu's threshold selection method [30] described in Appendix A. This histogram technique minimizes the within-class variance and maximizes the separation of classes, and thus gives an ideal solution to our problem. We have also tested another threshold selection method modeling the histograms with the sum of two Gaussian functions, however, numerically this proved to be less stable and less reliable.

The condition $S>S_{T H}$, made it possible to identify the grains in high shear intensity regions - where shear bands emerge - and thus the average volume fraction $\eta_{S B}$ of these regions could be calculated. The local packing density in shear bands is found to have small fluctuations and to give a peak in the volume fraction histograms. This coincides with $\eta_{S B}$ (see Fig. 2 (b, f)), giving a self validation of the method.

Let us note that even if Fig. 2 (e) does not suggest a clean separation of the grains into those within and those outside the shear bands - i.e. there is no shear intensity gap between the two regimes - this is not crucial for $\eta_{S B}$. Adding an artificial random noise of $10 \%$ to $S_{T H}$ does influence the resulting $\eta_{S B}$ only within $0.5 \%$.

We also mention here, that before strain localization takes place the shear intensity histograms have only one peak (see Fig. 2 (c)). In this case, the threshold given
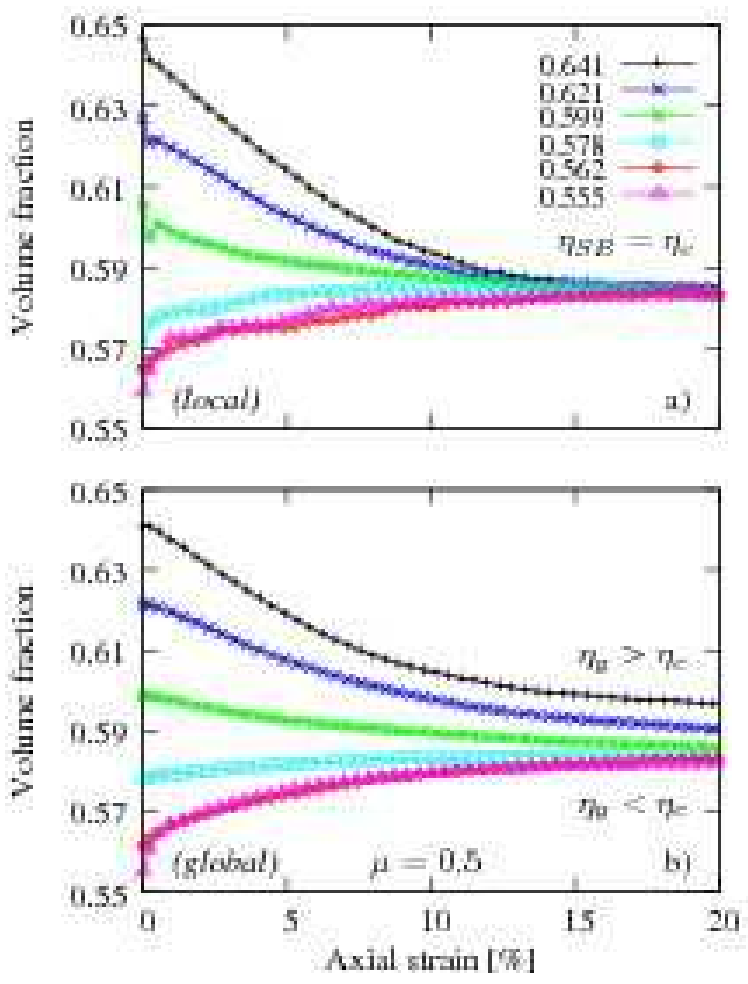

FIG. 3: (Color online) Volume fraction measured in high shear intensity regions (a) and globally (b) as function of the axial strain. The different lines correspond to different initial packing densities $\eta_{0}$ (see Tab. 【) decreasing from top to bottom. The two panels use the same notations.

by Otsu's method, which falls on the middle of the peak, is not physically relevant. However, as the sample is homogeneous, the selected samples in high shear intensity regions still give the volume fraction which is close to the average volume fraction of the whole sample. This can be verified on Fig. 2 (d).

\section{RESULTS}

As expected [12, 14], we found that due to strain localization at the end of the shear tests the global volume fraction of the samples is not equal to the packing density of the high shear intensity regions and thus global measurements cannot be used to characterize the properties of failure zones. The behavior of both dense and loose samples demonstrates that in the shear bands, the initial packing conditions are canceled and a critical volume fraction $\eta_{c}$ is reached in a self-organizing manner independently of the initial density of the tested granular specimens (Fig. 3 (a)).

The criticality is found to be restricted to the shear bands. The global volume fraction $\eta_{g}$ calculated from the total volume of the samples does not converge to $\eta_{c}$ (Fig. 3 (b)). This behavior is expected to be more pronounced on larger systems. The dense samples are 


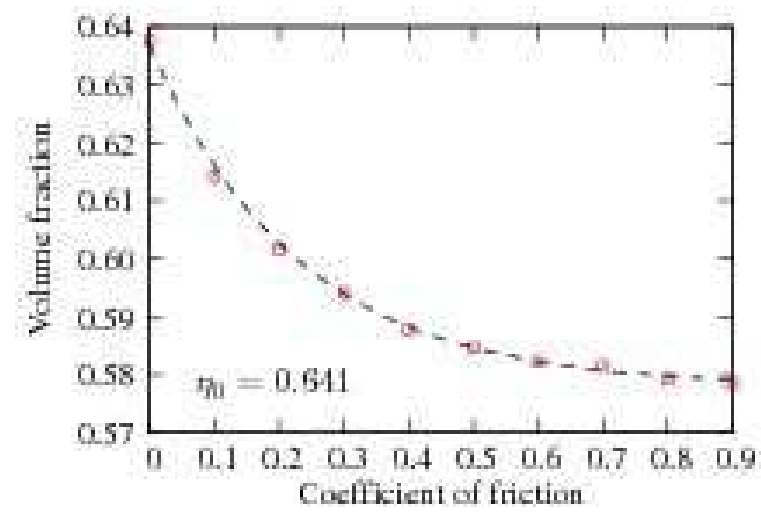

FIG. 4: (Color online) Critical packing of shear bands as function of friction measured at $20 \%$ axial strain. The fitted curve shows $\eta_{c}(\mu)=\eta_{c}^{\infty}-\left(\eta_{c}^{\infty}-\eta_{c}^{0}\right) \exp \left(-\mu / \mu_{c}^{0}\right)$, where $\eta_{c}^{0}=$ $0.637, \eta_{c}^{\infty}=0.578$, and $\mu_{c}^{0}=0.23$.

characterized by $\eta_{g}>\eta_{c}$. This demonstrates that the dilatancy [7] is concentrated to the shear bands. Contrary, for loose samples $\eta_{g}<\eta_{c}$, however, the specimen is only slightly looser outside the shear bands. This gives a direct proof of shear induced compaction [9].

As we could see, at given friction, the critical packing of shear bands is independent of the initial density. It is, however, a further question whether it depends on friction. We studied quantitatively this effect for samples with initial density $\eta_{0}=0.641$. At $\mu=0$ the high shear intensity regions have a large volume fraction $\eta_{c}^{0} \equiv \eta_{c}(0)=0.637 \pm 0.002$, which is only slightly smaller than $\eta_{0}$, showing that frictionless granular systems can be sheared at densities very close to the RCP limit.

In frictional systems, the volume fraction of the fully developed shear bands is substantially lower than the initial volume fraction (see Fig. 4). With increasing $\mu$, this decreases and converges to a limit which we estimate to $\eta_{c}^{\infty} \equiv \lim _{\mu \rightarrow \infty} \eta_{c}(\mu)=0.578 \pm 0.003$ based on the exponential extrapolation of our data.

This limit volume fraction depends only on geometry factors such as shape and size distribution of the grains and is characteristic to the dynamic equilibrium between dilation and compaction developed in a self-organized manner through strain localization. Based on the value of $\eta_{c}^{\infty}$ and this latter aspect, the corresponding asymptotic state - which we refer to as the dynamic random loose packing (DRLP) - should be distinguished from the static RLP limit.

\section{CONCLUSIONS AND DISCUSSION}

We have presented distinct element simulations of axisymmetric triaxial shear tests at zero gravity and low confining pressure. Due to spontaneous strain localization, shear bands were formed. Using a histogram technique, we identified the grains in high shear intensity regions, which at large axial strains coincide with the shear bands. We measured the packing density $\eta_{S B}$ inside these failure zones and we found that in fully developed shear bands $\eta_{S B}$ approaches a critical value $\eta_{c}$ independent of the initial density of the samples. This in agreement with Casagrande's [10] observation made for sandy soils seven decades before and also with recent experiments [12, 13, 14, 15] and numerical studies [17, 18, 19].

Rothenburg and Kruyt [18] obtained similar results in two-dimensional simulations of biaxial shear tests. They have presented a theory of the average coordination number of sheared granular media and derived a law for its evolution during slow deformations. Analyzing the relationship between volume fraction and average coordination number, they conclude that a proper characterization of granular media undergoing shear deformation should be based on packing density.

We have shown that the criticality is restricted to the shear bands and global measurements (such as dilatancy) are unsuitable for the investigation of the properties of sheared granular materials in realistic situations, where strain localization is inevitable. To our knowledge, it is the first time that the critical packing density of shear bands was evidenced based on simulations of a realistic three-dimensional setup and spontaneous strain localization revealing the self-organizing manner in which the packing state of the shear bands is developed.

We have further shown that $\eta_{c}$ depends on the coefficient of friction $\mu$ and in the limit $\mu \rightarrow \infty$ it converges to a value $\eta_{c}^{\infty}$, which we have calculated within the accuracy of our simulations. The found $\eta_{c}^{\infty}$ defines a low density dynamic random loose packing (DRLP) state, which is characteristic to the dynamic equilibrium between dilation and compaction in the shear bands and depends only on the geometry of the grains. Based on the underlying mechanism, we argue that the asymptotic packing state of shear bands differs from the static RLP limit.

This result should be also compared with the findings presented recently by Zhang and Makse [6] regarding the critical density of granular materials at jamming transition. The importance of these findings lie in the fact that jamming is a basic concept which through a unifying phase diagram [31, 32] connects granular matters with a variety of other systems including dense particulate suspensions and effects such as diverging viscosity at a maximum packing fraction [33, 34].

In quasistatic limit, Zhang and Makse [6] reported a monotonous decrease of the critical packing density as function of friction. For low friction, the density of the shear bands found in our simulations is lower than at jamming found by Zhang and Makse [6], while at high friction the situation is reversed. This indicates a natural separation of low and high density regions with possibly different mechanisms of dissolving the jammed state.

As a final remark let us note that our results are derived for idealized granular materials composed of spheres having a narrow size distribution. It is well known that for non-spherical grains and wide size distributions the packing efficiency increases [35], which should be also re- 
flected in the packing density of the shear bands. This could be the reason why experimental results on sand reveal smaller volume fractions in shear bands [12, 14] than the values found in our simulations.

\section{ACKNOWLEDGMENTS}

This research was carried out within the framework of the "Center for Applied Mathematics and Computational Physics" of the BME, and it was supported by OTKA F047259 and T049403, and the Péter Pázmány program RET-06/2005. S.F. thanks D. Chetverikov for the introduction to Otsu's method.

\section{APPENDIX A: OTSU'S THRESHOLD SELECTION METHOD}

Otsu's method [30] is a histogram technique known from Digital Image Processing, where it is typically used to transform grayscale images into two component (black and white) images.

Let us consider a normalized histogram $P(i)$, i.e. a histogram with the property

$$
\sum_{i} P(i)=1
$$

where $i$ is the bin index. The mean $\mu$ and the variance $\sigma^{2}$ can be calculated as

$$
\begin{aligned}
\mu & =\sum_{i} i P(i), \\
\sigma^{2} & =\sum_{i}(i-\mu)^{2} P(i) .
\end{aligned}
$$

Let us further consider a candidate threshold $t$ and split the histogram in two parts $\mathcal{I}_{1}(t)=\{i \mid i \leq t\}$ and $\mathcal{I}_{2}(t)=\{i \mid i>t\}$. With $k \in\{1,2\}$ and

$$
q_{k}(t)=\sum_{i \in \mathcal{I}_{k}(t)} P(i)
$$

the mean $\mu_{k}(t)$ and variance $\sigma_{k}^{2}(t)$ of the two parts are defined by the equations

$$
\begin{aligned}
q_{k}(t) \mu_{k}(t) & =\sum_{i \in \mathcal{I}_{k}(t)} i P(i), \\
q_{k}(t) \sigma_{k}^{2}(t) & =\sum_{i \in \mathcal{I}_{k}(t)}\left(i-\mu_{k}(t)\right)^{2} P(i) .
\end{aligned}
$$

The within-class variance

$$
\sigma_{W}^{2}(t)=q_{1}(t) \sigma_{1}^{2}(t)+q_{2}(t) \sigma_{2}^{2}(t)
$$

is an inverse measure of the compactness of classes. The between-class variance

$$
\sigma_{B}^{2}(t)=q_{1}(t) q_{2}(t)\left(\mu_{1}(t)-\mu_{2}(t)\right)^{2}
$$

is a measure of the separation of classes. It is easy to show that $\sigma_{W}^{2}(t)+\sigma_{B}^{2}(t)=\sigma^{2}$. Otsu [30] proposed to calculate an optimal threshold $t=t_{\text {opt }}$ by either minimizing $\sigma_{W}^{2}(t)$ or maximizing $\sigma_{B}^{2}(t)$.

Maximizing $\sigma_{B}^{2}(t)$ is easier. It can be seen that

$$
\begin{aligned}
q_{2}(t) & =1-q_{1}(t), \\
\mu_{2}(t) & =\frac{\mu-q_{1}(t) \mu_{1}(t)}{q_{2}(t)},
\end{aligned}
$$

and thus

$$
\sigma_{B}^{2}(t)=\frac{q_{1}(t)}{1-q_{1}(t)}\left(\mu_{1}(t)-\mu\right)^{2}
$$

For each candidate threshold $t, q_{1}(t)$ and $\mu_{1}(t)$ can be calculated with the recursive formula

$$
\begin{aligned}
q_{1}(t+1) & =q_{1}(t)+P(t+1) \\
\mu_{1}(t+1) & =\frac{q_{1}(t) \mu_{1}(t)+(t+1) P(t+1)}{q_{1}(t+1)}
\end{aligned}
$$

where $q_{1}(0)=P(0)$ and $\mu_{1}(0)=0$.

Both $q_{1}(t)$ and $\mu_{1}(t)$ are increasing monotonously with $t$, consequently the maximum of $\sigma_{B}^{2}(t)$ is well-defined, except for degenerated cases which must be handled separately. The optimal threshold $t_{\text {opt }}$ is given by the smallest candidate threshold $s$ which satisfies the equation

$$
\sigma_{B}^{2}(s)=\max _{t} \sigma_{B}^{2}(t)
$$

Because $\sigma_{W}^{2}\left(t_{\text {opt }}\right)+\sigma_{B}^{2}\left(t_{\text {opt }}\right)=\sigma^{2}$, the method both minimizes the within-class variance and maximizes the separation of classes.
[1] D. A. Weitz, Science 303, 968 (2004).

[2] T. Aste, M. Saadatfar, and T. J. Senden, Phys. Rev. E 71, 061302 (2005).

[3] S. Torquato, T. M. Truskett, and P. G. Debenedetti,
Phys. Rev. Lett. 84, 2064 (2000).

[4] C. S. O'Hern, L. E. Silbert, A. J. Liu, and S. R. Nagel, Phys. Rev. E 68, 011306 (2003).

[5] G. Y. Onoda and E. G. Liniger, Phys. Rev. Lett. 64, 
2727 (1990).

[6] H. P. Zhang and H. A. Makse, Phys. Rev. E 72, 011301 (2005), and references therein.

[7] O. Reynolds, Philos. Mag. 20, 469 (1885).

[8] J. Török, S. Krishnamurthy, J. Kertész, and S. Roux, Phys. Rev. Lett. 84, 3851 (2000).

[9] T. L. Youd, Journal of the Soil Mechanics and Foundations Division 98, 709 (1972).

[10] A. Casagrande, Journal of the Boston Society of Civil Engineers 23, 257 (1936).

[11] A. N. Schofield and P. Wroth, Critical State Soil Mechanics (McGraw-Hill, London, 1968).

[12] J. Desrues, in X-ray CT for Geomaterials, edited by J. Otani and Y. Obara (Balkema, Amsterdam, 2004), pp. $15-41$.

[13] J. Desrues, R. Chambon, M. Mokni, and F. Mazerolle, Géotechnique 46, 529 (1996).

[14] S. N. Batiste, K. A. Alshibli, S. Sture, and M. Lankton, Geotechnical Testing Journal 27, 568 (2004).

[15] K. A. Alshibli, S. Sture, N. C. Costes, M. L. Frank, M. R. Lankton, S. N. Batiste, and R. A. Swanson, Geotechnical Testing Journal 23, 274 (2000).

[16] T. Pöschel and T. Schwager, Computational Granular Dynamics: Models and Algorithms (Springer, Berlin, 2005), and references therein.

[17] M. Piccioni, V. Loreto, and S. Roux, Phys. Rev. E 61, 2813 (2000).

[18] L. Rothenburg and N. P. Kruyt, International Journal of Solids and Structures 41, 5763 (2004).

[19] X. Zhuang, A. K. Didwania, and J. D. Goddard, Journal of Computational Physics 121, 331 (1995).

[20] S. Fazekas, J. Török, J. Kertész, and D. E. Wolf, Phys. Rev. E 74, 031303 (2006), cond-mat/0506661.

[21] P. A. Cundall and O. D. L. Strack, Geotechnique 29, 47 (1979), and articles citing this one.

[22] L. D. Landau and E. M. Lifshitz, Theory of Elasticity (Pergamon, New York, 1970), chap. 9, 2nd ed.
[23] S. Fazekas, J. Török, J. Kertész, and D. E. Wolf, in Powders and Grains 2005, edited by R. García-Rojo, H. J. Herrmann, and S. McNamara (Balkema, London, 2005), pp. 223-226, cond-mat/0606720.

[24] H. Tsunekawa and K. Iwashita, in Powders and Grains 2001, edited by Y. Kishino (Balkema, Rotterdam, 2001), pp. $177-180$.

[25] H. Sakaguchi and H.-B. Mühlhaus, in Exploration Geodynamics Chapman Conference (American Geophysical Union, Dunsborough, Western Australia, 2001), pp. 153155.

[26] L. Cui and C. O'Sullivan, in Powders and Grains 2005, edited by R. García-Rojo, H. J. Herrmann, and S. McNamara (Balkema, London, 2005), pp. 301-305.

[27] C. Lee, in Applied Geometry and Discrete Mathematics: The Victor Klee Festschrift, edited by P. Gritzmann and B. Sturmfels (American Mathematical Society, Providence, RI, 1991), pp. 443-456.

[28] H. Edelsbrunner and N. R. Shah, Algorithmica 15, 223 (1996), and references therein.

[29] D. Daudon, J. Lanier, and M. Jean, in Powders and Grains 1997, edited by R. P. Behringer and J. T. Jenkins (Balkema, London, 1997), pp. 219-222.

[30] N. Otsu, IEEE Trans. Systems, Man and Cybernetics 9, 62 (1979).

[31] V. Trappe, V. Prasad, L. Cipelletti, P. N. Segre, and D. A. Weitz, Nature 411, 773 (2001).

[32] A. J. Liu and S. R. Nagel, Nature 396, 21 (1998).

[33] J. J. Stickel and R. L. Powell, Annual Review of Fluid Mechanics 37, 129 (2005).

[34] G. Ovarlez, F. Bertrand, and S. Rodts, Journal of Rheology 50, 259 (2006), and references therein.

[35] A. Donev, I. Cisse, D. Sachs, E. A. Variano, F. H. Stillinger, R. Connelly, S. Torquato, and P. M. Chaikin, Science 303, 990 (2004). 